\title{
Ascospore Infection and Colletotrichum Species Causing Glomerella Leaf Spot of Apple in Uruguay
}

\author{
Sandra Alaniz (i) ${ }^{1 *}$, Vanessa Cuozzo ${ }^{1}$, Valentina Martínez ${ }^{1}$, Marciel J. Stadnik ${ }^{2}$, and Pedro Mondino ${ }^{1}$ \\ ${ }^{I}$ Department of Plant Protection, Faculty of Agronomy, University of the Republic, Avenue Garzón 780, Montevideo \\ 12900, Uruguay \\ ${ }^{2}$ Laboratory of Plant Pathology, Agricultural Science Center, Federal University of Santa Catarina, Road Admar Gon- \\ zaga 1346, Florianopolis-SC 88034-001, Brazil
}

(Received on July 31, 2018; Revised on December 5, 2018; Accepted on December 17, 2018)

Glomerella leaf spot (GLS) caused by Colletotrichum spp. is a destructive disease of apple restricted to a few regions worldwide. The distribution and evolution of GLS symptoms were observed for two years in Uruguay. The recurrent ascopore production on leaves and the widespread randomized distribution of symptoms throughout trees and orchard, suggest that ascospores play an important role in the disease dispersion. The ability of ascospores to produce typical GLS symptom was demonstrated by artificial inoculation. Colletotrichum strains causing GLS did not result in rot development, despite remaining alive in fruit lesions. Based on phylogenetic analysis of actin, $\beta$-tubulin and glyceraldehyde-3-phosphate dehydrogenase gene regions of $\mathbf{4 6}$ isolates, 25 from fruits and 21 from leaves, C. karstii was identified for the first time causing GLS in Uruguay and $C$. fructicola was found to be the most frequent (89\%) and aggressive species. The higher aggressiveness of $\boldsymbol{C}$. fructicola and its ability on to produce abundant fertile perithecia could help to explain the predominance of this species in the field.

Keywords : Colletotrichum fructicola, Colletotrichum

\author{
*Corresponding author. \\ Phone) +598-23551108, FAX) +598-23563096 \\ E-mail)salaniz@fagro.edu.uy \\ ORCID \\ Sandra Alaniz \\ http://orcid.org/0000-0002-6530-7279 \\ (c) This is an Open Access article distributed under the terms of the \\ Creative Commons Attribution Non-Commercial License (http:// \\ creativecommons.org/licenses/by-nc/4.0) which permits unrestricted \\ noncommercial use, distribution, and reproduction in any medium, \\ provided the original work is properly cited.
}

Articles can be freely viewed online at www.ppjonline.org. karstii, Malus

Handling Editor : Kim, Ki Woo

Glomerella leaf spot (GLS) is a relatively recent and destructive disease of apple, but still restricted to a few world regions. GLS was reported for the first time in the Southwest of USA in 1970 (Taylor, 1971) but only at the end of the 90s became more important when a severe outbreak was reported (Gonzalez and Sutton, 1999). In 1983, GLS appeared in Southern Brazil, and since then it has been considered one of the major diseases of apple (Sutton and Sanhueza, 1998). In 2012, it was reported in Eastern China as a new destructive disease of apple (Wang et al., 2012). Later on, in 2016 it was noticed in Uruguay causing severe defoliation in a commercial apple orchard located in the Southern part of the country (Casanova et al., 2017).

The symptoms of GLS appear during the summer on leaves and fruits of apple. On leaves they consist in brown to ochre necrotic spot lesions that grow quickly taking an irregular shape and causing generalized chlorosis. Under warm and hot conditions, severe defoliation of the affected tree can be observed. On fruits, circular, slightly sunken and corky lesions of 0.5 to $3 \mathrm{~mm}$ in diameter and 0.5 to 2 $\mathrm{mm}$ in deep appear; these lesions are usually surrounded by a red border (Casanova et al., 2017; González and Sutton, 1999, 2004; Wang et al., 2012). Cultivars as Golden Delicious and their descendants such as cv. Galaxy or Cripps pink are highly susceptible, but others like Red Delicious and Fuji are highly resistant (Liu et al., 2016; Velho et al., 2015; Wang et al., 2015b). In Uruguay, commercial apple production is concentrated in the South where $22 \%$ of orchard trees belong to cvs. Galaxy and Cripps pink (MGAPDIEA, 2016). 
Phenotypic analyses initially identified Glomerella cingulata, Colletotrichum gloeosporioides and C. acutatum as the causal agents of GLS in Brazil and USA (González and Sutton, 1999, 2004; Sutton and Sanhueza, 1998) and to $G$. cingulata in China (Wang et al., 2012). Later, using multilocus genetic markers the species associated to GLS in Brazil were reidentified as $C$. fructicola (Velho et al., 2015) and C. karstii (Velho et al., 2014) and C. fructicola and C. aegnima in China (Wang et al., 2015b).

GLS has gradually spread to new apple production regions, increasing its economic impact worldwide. However, partially due to its recent emergence, only a few studies have been carried out so far, particularly those focusing on epidemiology and control. Acervuli and perithecia containing conidia and ascospores are produced in lesions of infected leaves (Casanova et al., 2017; Valdebenito-Sanuheza et al., 2002), but the role of both types of spores in the development of the disease, is poorly understood. Pathogenicity tests with leaves and fruits by means of artificial inoculation with conidial suspensions of Colletotrichum spp. have been successfully conducted (Casanova et al., 2017; Velho et al., 2014, 2015; Wang et al., 2015b). In contrast, the ability of ascospores in producing symptoms of GLS on both leaves and fruits has not been demonstrated.

In order to better understand the disease occurrence in Uruguay, the objectives of our study were i) monitor the distribution and evolution of GLS symptoms on commercial apple orchards ii) identify Colletotrichum species involved with GLS symptoms in fruit and leaves, and iii) test the ability of ascospores in causing GLS symptoms on apple leaves and fruits.

\section{Materials and Methods}

GLS field symptoms and fungal isolates. In summer 2015 and 2016, the presence of symptoms and their distribution were observed in trees of the GLS-diseasedorchards composed of cv. Cripps pink with cv. Galaxy as pollinator in Kiyu, San José Department $\left(34^{\circ} 38^{\prime *} \mathrm{~S}^{*} 56^{\circ}\right.$ $\left.45^{\prime *} \mathrm{~W}^{*}\right)$, Uruguay. Leaves and fruits with typical GLS symptoms were collected from trees randomly selected; a maximum of three samples per tree including fruits and leaves were taken.

To isolate the fungus from the lesions, the fruits were first disinfected with $70 \%$ ethanol. After drying, fruit peel was removed from the affected area with a sterile scalpel leaving the internal lesion exposed. Small portions of the corky lesion were plated in potato dextrose agar (PDA) (Oxoid Ltd., Hampshire, England) and incubated at $25^{\circ} \mathrm{C}$ in the dark for 5 days. Hyphae growing out from fruit tissues were subcultured onto PDA media.

Monoascospore and monoconidial isolates were obtained directly from leaf lesions under magnifying glass. For this, a single perithecium or acervulus was transferred from leaf lesions to a tube containing $300 \mu \mathrm{l}$ of sterile distilled water (SDW) (one per leaf) and vigorously stirred using a vortex. Immediately, $100 \mu 1$ of ascospores or conidial suspension was placed onto PDA supplemented with $0.4 \mathrm{~g} / 1$ of streptomycin sulphate (Sigma-Aldrich, China). After $24 \mathrm{~h}$ of incubation at $25^{\circ} \mathrm{C}$ in the dark, a germinated ascospore or conidium was transferred to PDA medium and incubated to the same conditions.

The Colletotrichum spp. isolates were stored at $-20^{\circ} \mathrm{C}$ in colonized sterile paper filters dried with silica-gel (Peever et al., 1999). Isolates were deposited in the culture collection of the Department of Plant Protection, Faculty of Agronomy, University of the Republic Uruguay.

Morphological characterization. All Colletotrichum isolates were grown on PDA medium for two to four weeks at $25^{\circ} \mathrm{C}$ and $12 \mathrm{~h}$ photoperiod under near ultraviolet light. Macroscopic characteristics, such as colony colour and presence of reproductive structures as perithecia, acervuli, asci, ascospores and conidia were recorded.

PCR amplification, sequencing and phylogenetic analysis. Mycelium and reproductive structures from all isolates grown on PDA for one week at $25^{\circ} \mathrm{C}$ in the dark were scraped off with a spatula and placed in $2.0 \mathrm{ml}$ microtubes. Total DNA was extracted using a protocol described by Paolocci et al. (1999).

The partial actin (ACT), $\beta$-tubulin (BTUB2) and glyceraldehyde-3-phosphate dehydrogenase (GAPDH) gene regions were amplified and sequenced for all Colletotrichum sp. isolates. The primer pairs used were ACT-512F/ACT-783R (Carbone and Kohn, 1999), BT2Fd/BT4R (Woudenberg et al., 2009) and GDF1/GDR1 (Guerber et al., 2003) respectively.

Each PCR reaction contained 1x PCR buffer, $2.5 \mathrm{mM}$ $\mathrm{MgCl}_{2}, 400 \mu \mathrm{M}$ of each dNTP, $0.4 \mu \mathrm{M}$ of each primer, 1 $\mathrm{U}$ of DNA polymerase (Tiangen, China) and $1 \mu \mathrm{l}$ of template DNA. The PCR reaction mix was adjusted to a final volume of $40 \mu \mathrm{l}$ with MQ water. The amplifications were performed on a MultiGene ${ }^{\mathrm{TM}}$ Mini (Labnet International, Inc., USA). The program run was as follows: an initial step of $94^{\circ} \mathrm{C}$ for $5 \mathrm{~min}$ followed by 34 cycles of denaturation at $94^{\circ} \mathrm{C}$ for $45 \mathrm{~s}$, annealing at $52^{\circ} \mathrm{C}\left(57^{\circ} \mathrm{C}\right.$ for ITS $)$ for 30 $\mathrm{s}$, and elongation at $72^{\circ} \mathrm{C}$ for $45 \mathrm{~s}$. A final extension was performed at $72^{\circ} \mathrm{C}$ for $10 \mathrm{~min}$. The PCR products were analyzed in $1.5 \%$ agarose gels stained with $\mathrm{GelRed}^{\mathrm{TM}}$ and 
visualized under UV light. A gene ruler 100-bp DNA ladder plus was used as a molecular weight marker (Thermo, Lithuania).

The PCR products were purified and sequenced in Macrogen Inc., Seoul, Korea. The DNA sequences were edited using DNAman 8.0 program (Lynnon Corporation). The sequences were aligned using ClustalW program (Larkin et al., 2007), available within MEGA 7.0.26 program (Kumar et al., 2016). The sequences derived from this study were deposited in the GenBank (Table 1). In the alignments,

Table 1. Isolates of Colletotrichum, host and cultivar, isolation organ, origin and GenBank accession no. of isolates of apple generated in this study and obtained from GenBank

\begin{tabular}{|c|c|c|c|c|c|c|c|}
\hline \multirow{2}{*}{ Specie } & \multirow{2}{*}{ Isolate } & \multirow{2}{*}{ Host, cultivar } & \multirow{2}{*}{ Organ } & \multirow{2}{*}{ Country } & \multicolumn{3}{|c|}{ GenBank accession no. } \\
\hline & & & & & Actin & BTUB2 & GAPDH \\
\hline C. acutatum & CBS 112996 & Carica papaya & & Australia & JQ005839 & JQ005860 & JQ948677 \\
\hline \multirow[t]{2}{*}{ C. aenigma } & C1253.4 & Persea americana & & Israel & JX009443 & JX010389 & JX010044 \\
\hline & C1253.6 & Pyrus piryfolia & & Japan & JX009519 & JX010390 & JX009913 \\
\hline C. annellatum & CBS 129826 & Hevea indica & leaf & Colombia & JQ005570 & JQ005656 & JQ005309 \\
\hline \multirow[t]{2}{*}{ C. alienum } & C824 & Malus domestica & & New Zealand & JX009572 & JX010411 & JX010028 \\
\hline & C1194.22 & Persea americana & & New Zealand & JX009552 & JX010386 & JX009959 \\
\hline \multirow[t]{33}{*}{ C. fructicola } & C1275.7 & Ficus edulis & & Germany & JX009495 & JX010400 & JX009923 \\
\hline & C1276.4 & Dioscorea alata & & Nigeria & JX009436 & JX010401 & JX010041 \\
\hline & L48* & Malus domestica, Galaxy & leaf & Uruguay & MG491754 & MG491713 & MG491672 \\
\hline & L49* & Malus domestica, Galaxy & leaf & Uruguay & MG491755 & MG491714 & MG491673 \\
\hline & L52* & Malus domestica, Galaxy & leaf & Uruguay & MG491756 & MG491715 & MG491674 \\
\hline & L53* & Malus domestica, Galaxy & leaf & Uruguay & MG491757 & MG491716 & MG491675 \\
\hline & L54* & Malus domestica, Galaxy & leaf & Uruguay & MG491758 & MG491717 & MG491676 \\
\hline & L56* & Malus domestica, Galaxy & leaf & Uruguay & MG491759 & MG491718 & MG491677 \\
\hline & L57* & Malus domestica, Galaxy & leaf & Uruguay & MG491760 & MG491719 & MG491678 \\
\hline & L58* & Malus domestica, Galaxy & leaf & Uruguay & MG491761 & MG491720 & MG491679 \\
\hline & L60* & Malus domestica, Galaxy & leaf & Uruguay & MG491762 & MG491721 & MG491680 \\
\hline & L61* & Malus domestica, Cripss pink & leaf & Uruguay & MG491763 & MG491722 & MG491681 \\
\hline & L63* & Malus domestica, Cripss pink & leaf & Uruguay & MG491764 & MG491723 & MG491682 \\
\hline & L64* & Malus domestica, Cripss pink & leaf & Uruguay & MG491765 & MG491724 & MG491683 \\
\hline & L66* & Malus domestica, Galaxy & leaf & Uruguay & MG491766 & MG491725 & MG491684 \\
\hline & L67* & Malus domestica, Galaxy & leaf & Uruguay & MG491767 & MG491726 & MG491685 \\
\hline & L68* & Malus domestica, Galaxy & leaf & Uruguay & MG491768 & MG491727 & MG491686 \\
\hline & L69* & Malus domestica, Galaxy & leaf & Uruguay & MG491769 & MG491728 & MG491687 \\
\hline & F70* & Malus domestica, Galaxy & fruit & Uruguay & MG491770 & MG491729 & MG491688 \\
\hline & F71* & Malus domestica, Galaxy & fruit & Uruguay & MG491771 & MG491730 & MG491689 \\
\hline & F72* & Malus domestica, Galaxy & fruit & Uruguay & MG491772 & MG491731 & MG491690 \\
\hline & F73* & Malus domestica, Galaxy & fruit & Uruguay & MG491773 & MG491732 & MG491691 \\
\hline & F74* & Malus domestica, Galaxy & fruit & Uruguay & MG491774 & MG491733 & MG491692 \\
\hline & F75* & Malus domestica, Galaxy & fruit & Uruguay & MG491775 & MG491734 & MG491693 \\
\hline & F76* & Malus domestica, Galaxy & fruit & Uruguay & MG491776 & MG491735 & MG491694 \\
\hline & F78* & Malus domestica, Galaxy & fruit & Uruguay & MG491777 & MG491736 & MG491695 \\
\hline & F79* & Malus domestica, Galaxy & fruit & Uruguay & MG491778 & MG491737 & MG491696 \\
\hline & F80* & Malus domestica, Galaxy & fruit & Uruguay & MG491779 & MG491738 & MG491697 \\
\hline & $\mathrm{F} 81^{*}$ & Malus domestica, Galaxy & fruit & Uruguay & MG491780 & MG491739 & MG491698 \\
\hline & $\mathrm{F} 82^{*}$ & Malus domestica, Galaxy & fruit & Uruguay & MG491781 & MG491740 & MG491699 \\
\hline & F83* & Malus domestica, Galaxy & fruit & Uruguay & MG491782 & MG491741 & MG491700 \\
\hline & F84* & Malus domestica, Galaxy & fruit & Uruguay & MG491783 & MG491742 & MG491701 \\
\hline & F85* & Malus domestica, Cripss pink & fruit & Uruguay & MG491784 & MG491743 & MG491702 \\
\hline
\end{tabular}


Table 1. Continued

\begin{tabular}{|c|c|c|c|c|c|c|c|}
\hline \multirow{2}{*}{ Specie } & \multirow{2}{*}{ Isolate } & \multirow{2}{*}{ Host, cultivar } & \multirow{2}{*}{ Organ } & \multirow{2}{*}{ Country } & \multicolumn{3}{|c|}{ GenBank accession no. } \\
\hline & & & & & Actin & BTUB2 & GAPDH \\
\hline & F86* & Malus domestica, Cripss pink & fruit & Uruguay & MG491785 & MG491744 & MG491703 \\
\hline & $\mathrm{F} 87^{*}$ & Malus domestica, Cripss pink & fruit & Uruguay & MG491786 & MG491745 & MG491704 \\
\hline & F88* & Malus domestica, Cripss pink & fruit & Uruguay & MG491787 & MG491746 & MG491705 \\
\hline & F89* & Malus domestica, Cripss pink & fruit & Uruguay & MG491788 & MG491747 & MG491706 \\
\hline & F90* & Malus domestica, Cripss pink & fruit & Uruguay & MG491789 & MG491748 & MG491707 \\
\hline & F91* & Malus domestica, Cripss pink & fruit & Uruguay & MG491790 & MG491749 & MG491708 \\
\hline & F92* & Malus domestica, Cripss pink & fruit & Uruguay & MG491791 & MG491750 & MG491709 \\
\hline & F93* & Malus domestica, Cripss pink & fruit & Uruguay & MG491792 & MG491751 & MG491710 \\
\hline & F94* & Malus domestica, Cripss pink & fruit & Uruguay & MG491793 & MG491752 & MG491711 \\
\hline & F95* & Malus domestica, Cripss pink & fruit & Uruguay & MG491794 & MG491753 & MG491712 \\
\hline \multirow[t]{8}{*}{ C. karstii } & CORCG6 & Vanda sp. & & China & HM581995 & HM585428 & HM585391 \\
\hline & CBS 112982 & Protea cynaroides & & Zimbawe & JQ005531 & JQ005617 & JQ005270 \\
\hline & BRI P28443a & Magnifera indica & & Australia & JQ005551 & JQ005637 & JQ005290 \\
\hline & L51* & Malus domestica, Galaxy & leaf & Uruguay & MG491805 & MG491800 & MG491795 \\
\hline & L55* & Malus domestica, Galaxy & leaf & Uruguay & MG491806 & MG491801 & MG491796 \\
\hline & L59* & Malus domestica, Galaxy & leaf & Uruguay & MG491807 & MG491802 & MG491797 \\
\hline & L62* & Malus domestica, Cripss pink & leaf & Uruguay & MG491808 & MG491803 & MG491798 \\
\hline & L65* & Malus domestica, Galaxy & leaf & Uruguay & MG491809 & MG491804 & MG491799 \\
\hline \multirow[t]{3}{*}{ C. nupharicola } & $\mathrm{C} 1275.24$ & Nupharlutea & & USA & JX009486 & JX010397 & JX009936 \\
\hline & C1275.25 & Nupharlutea & & USA & JX009437 & JX010398 & JX009972 \\
\hline & $\mathrm{C} 1275.26$ & Nymphaea ordorata & & USA & JX009582 & JX010399 & JX010031 \\
\hline \multirow[t]{2}{*}{ C. petchi } & CBS 378.94 & Dracaena marginata & leaf & Italy & JQ005571 & JQ005657 & JQ005310 \\
\hline & CBS 118193 & Dracaena sanderana & leaf & China & JQ005575 & JQ005661 & JQ005314 \\
\hline C. phyllanthi & CBS175.67 & Phyllanthus acidus & & India & JQ005569 & JQ005655 & JQ005308 \\
\hline
\end{tabular}

Ex-types isolates are indicated as bold bold

*Indicate isolates generated in this study

related sequences and sequences of the phylogenetically closest species (including ex-type) of $C$. boninense and $C$. gloeosporioides species complexes obtained from the GenBank, were incorporated (Damm et al., 2012; Weir et al., 2012). The ex-type of C. acutatum CBS 112996 was used as the out-group. The sequence alignment was deposited in TreeBASE (www.treebase.org) under the accession number S21955.

Phylogenetic analyses were performed for each gene region separately as well as for the multi-locus alignment using MEGA 7.0.26 program for maximum likelihood (ML) and maximum parsimony (MP) analysis. To confirm that the three sequence datasets were congruent and combinable, highly supported clades among the trees generated from the different data sets for MP and ML analysis were compared to detect conflicts. High support typically refers to bootstrap support values $\geq 70 \%$ (Hillis and Bull, 1993).

ML analysis was based on the Tamura-Nei model and the tree was drawn to scale, with branch lengths measured in the number of substitutions per site. MP analysis was performed using the subtree pruning regrafting (SPR) algorithm with search level 1, in which the initial trees were obtained with random additions of sequences (10 replicates). All characters were unordered and of equal weight. Gaps were treated as missing data. The tree length (TL), consistency index $(\mathrm{CI})$, and retention index (RI) were recorded. The robustness of the the ML and MP trees was assessed by bootstrap analysis with 1,000 replicates (Hillis and Bull, 1993).

Pathogenicity tests. The pathogenicity of the isolate F51 of C. karstii was tested with conidia suspension, whereas the perithecial isolates L49, L53, L64, F71 and F84 of C. fructiola were used to verify the ability of ascospores in causing GLS symptoms. The six strains were randomly selected and tested against both apple fruits and leaves.

Isolates were grown on PDA at $25^{\circ} \mathrm{C}$ for three weeks with a $12 \mathrm{~h}$ photoperiod under near ultra violet light to induce sporulation. To prepare ascospore suspensions, perithecia were removed with sterile fine forceps from 

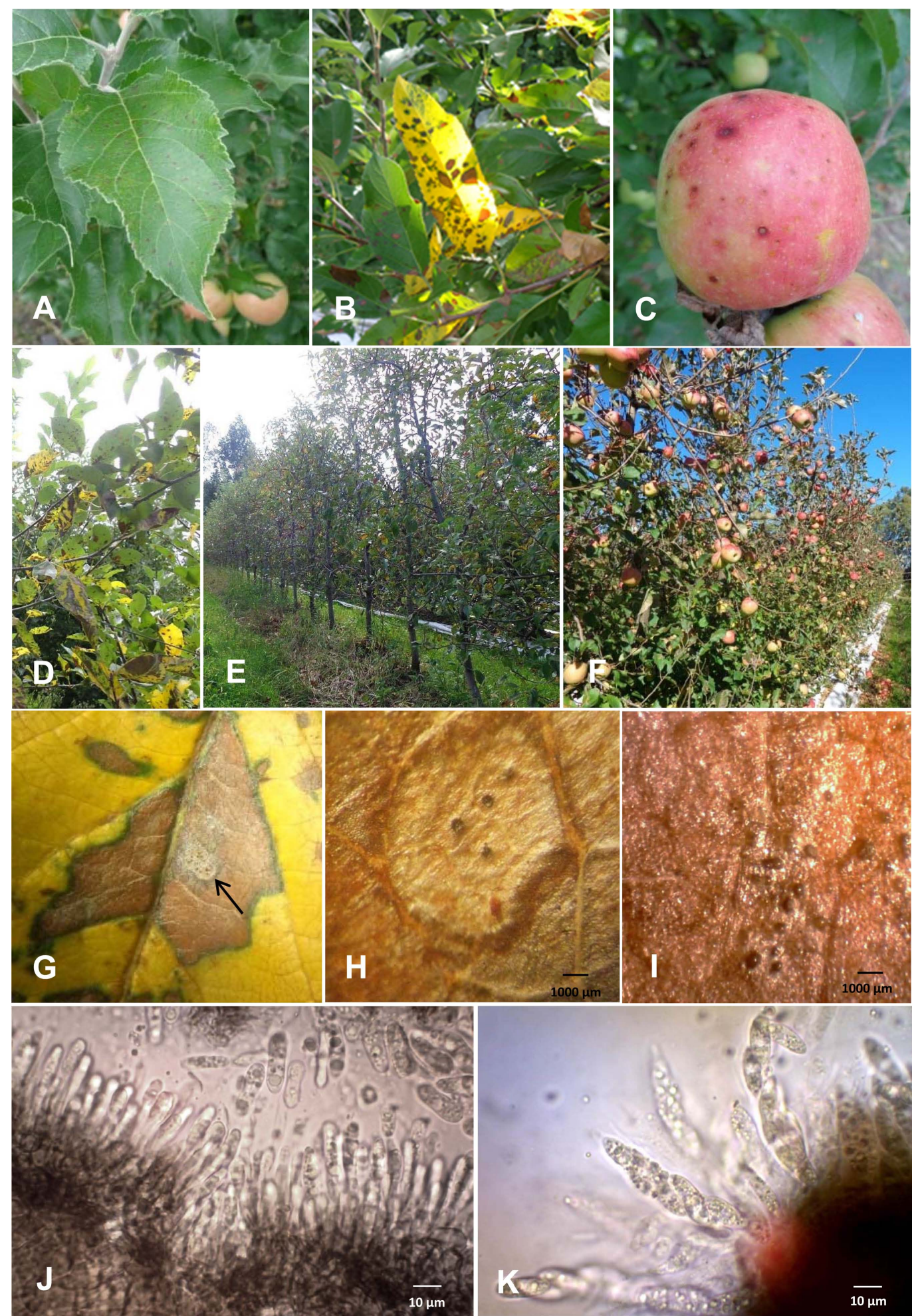

Fig. 1. Symptoms of Glomerella leaf spot (GLS) on apple. (A) Initial brown to ochre necrotic leaf lesions and (B) advanced, irregular and necrotic leaf lesions associated to chlorosis. (C) Fruit lesions surrounded by a red border. (D) Random distribution of leaf lesions in the tree. (E) Random distribution of symptoms in the orchards and severe defoliation in cv. Galaxy and (F) cv. Cripps pink. (G) Reproductive structures of Colletotrichum spp. over leaf lesion (black arrow). Structures present on leaves with typical symptoms of Glomerella leaf spot disease, collected from apple orchards. Detail of (H) acervuli and (I) perithecia. Micrsocopic detail of crushed (J) acervulus showing conidiphore and conida and $(\mathrm{K})$ peritecium showing asci and ascospores. 
the colonies of $C$. fructicola growing on PDA, placed into sterile tube of $2 \mathrm{ml}$ with SDW and shortly vortexed during one minute. To prepare the conidial suspension, colony of $C$. karstii growing on PDA was flooded with $10 \mathrm{ml}$ of SDW and then scraped with a sterile spatule. In both cases, the resulting suspension was filtered through two layers of cheese cloth and adjusted with a counting chamber to $1 \times$ $10^{5}$ ascospores or conidia $/ \mathrm{ml}$.

Young terminal twigs of cv. Galaxy were collected from a disease-free commercial apple orchard. For the first assay, fully developed young leaves were selected and placed individually in Petri dishes containing moistened sterile paper filter. To avoid the dehydration, the petiole of each leaf was wrapped with wet cotton. The detached leaves were inoculated by spraying (three leaves per isolate) and then incubated at $25^{\circ} \mathrm{C}$. Control leaves were spraying with SDW.

For the second assay, another set of young terminal twigs with 5-6 leaves were planted individually in glass flasks containing wet sand. The twigs were inoculated by spraying the spore suspension (five twigs per isolate). They were immediately covered with a moistened plastic bag and incubated at $25^{\circ} \mathrm{C}$. Control twigs were spraying with SDW.

Asymptomatic apple fruits of the cv. Cripps pink at the beginning of colour change were harvested from a diseasefree commercial orchard. All fruits were surface-disinfested with $70 \%$ ethanol. After that, fruits were inoculated by spraying (five per treatment) and incubated in moistened plastic bags at $25^{\circ} \mathrm{C}$. Control fruits were spraying with SDW.
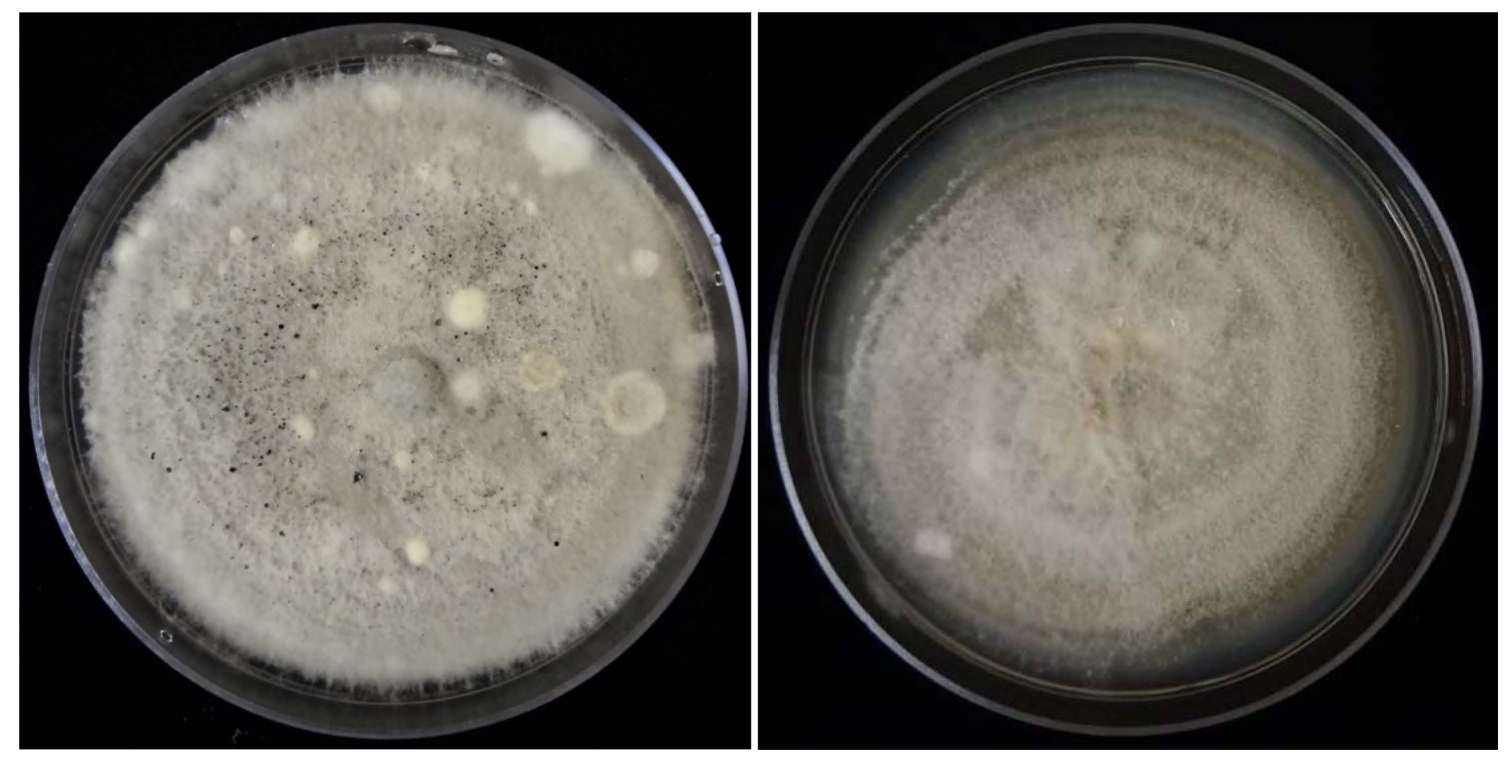

Fig. 2. Colony morphology of Colletotrichum fructicola isolate F71 (right) with abundant black perithecia and of C. karstii isolate L51 (left) after three weeks growing on PDA.

All assays were monitored daily; the initial symptoms and their evolution as well the presence of reproductive structures of Colletotrichum were recorded. C. karstii was reisolated from leaves and fruits to complete Koch's postulates.

\section{Results}

GLS field symptoms and fungal isolates. Typical GLS symptoms (Casanova et al., 2017) were observed on leaves and fruits in both years and on both apple cvs., Cripps pink and Galaxy. The first GLS disease symptoms were observed during January, primarily occurring in leaves (Fig. 1A). The leaf lesions rapidly evolved into chlorosis (Fig. 1B) and, approximately one month later, a severe defoliation (approximately $50 \%$ of fallen leaves) was observed (Fig. 1E and 1F). The fruit lesions did not evolve into rot remaining as small corky lesions (Fig. 1C).

The spatial distribution of symptoms was widespread randomized within orchards as well as individual trees. In each apple tree, infected leaves and fruits were observed at different heights, even in the lower inner tree canopy and terminal twigs (Fig. 1D). Presence of acervuli (Fig. 1H) and perithecia (Fig. 1I) containing conidia and ascospores, respectively, were consistently observed in leaf lesions (Fig. $1 \mathrm{G})$, but never in fruit lesions.

Typical colonies of the Colletotrichum fungus, i.e. showing white to orange or white to gray mycelium colour, presence of orange-salmon gelatinous masses composed of hyaline, fusiform to obtuse conidia, successfully grew from

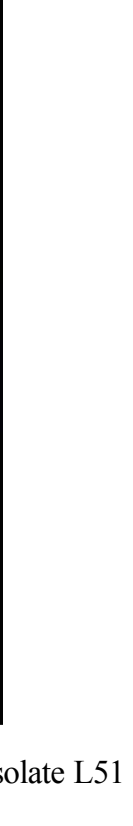




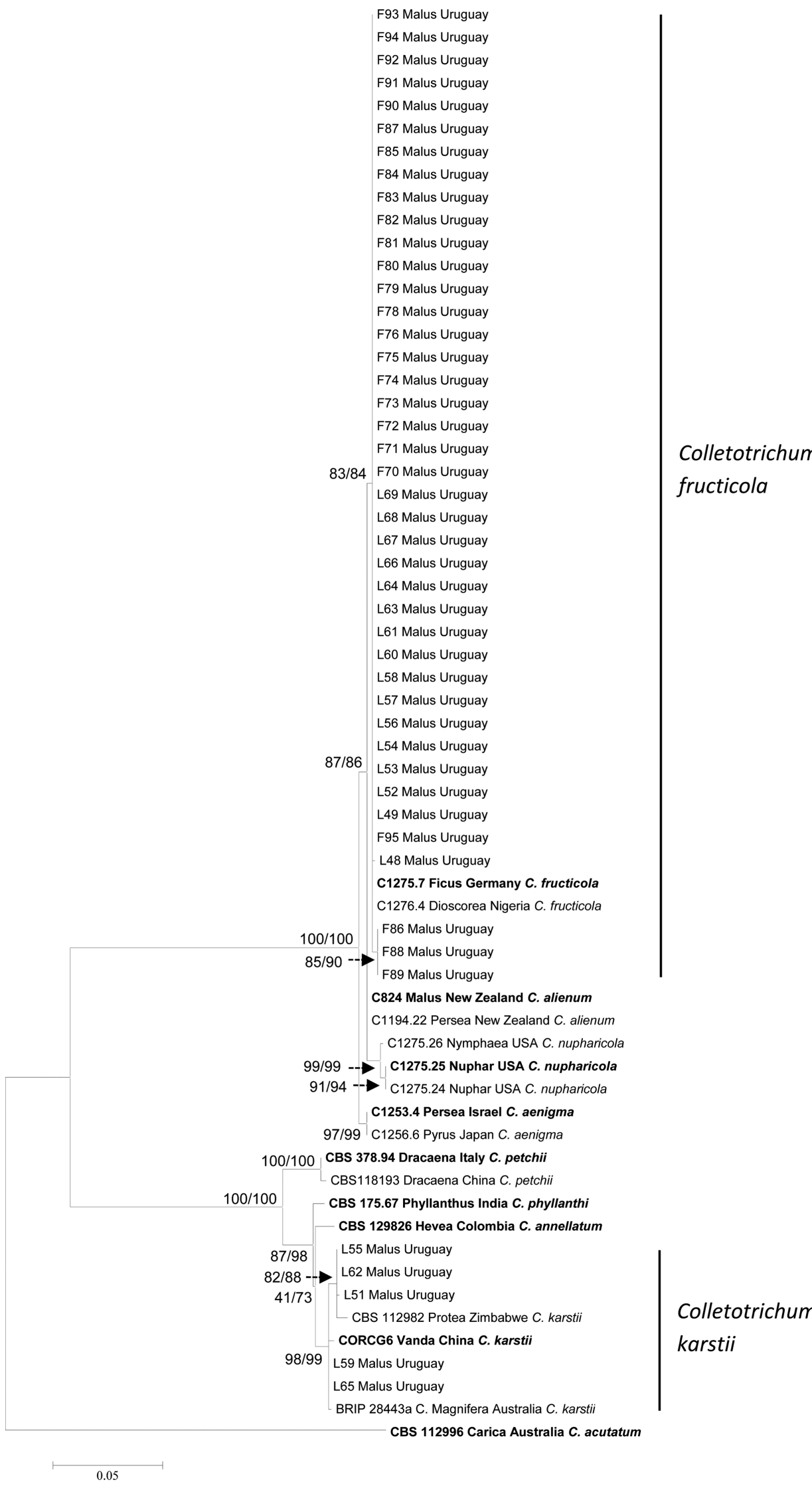

Fig. 3. Maximum likelihood phylogenetic tree generated from conctenated actine, $\beta$-tubulin and glyceraldehyde3 -phosphate dehydrogenase sequences alignment of 46 Uruguayan isolates obtained from leaf (indicated with L) and fruit (indicated with F) of apple with GLS (Glomerella Leaf Spot) symptoms. Sequences of the representative species of Colletotrichum boninense and C. gloeosporiodes species complexes extracted from GenBank (ex-type are indicated as bold) were included. C. acutatum CBS 112996 was used as the outgroup. Bootstrap support values of maximum likelihood and maximum parsimony are shown at the nodes before and after the bar, respectively. 
fruit and leaf infected tissues. Forty-six isolates of Colletotrichum were obtained, 21 originated from leaves and 25 from fruits (Table 1).

Morphological characterization. Initially the colony color of all the isolates on PDA was predominately white, but a few days later, they gradually changed to pale-gray to gray.

Reproductive structures were observed in all colonies. For the strains phylogenticaly identified as C. fructicola, the predominating structures were perithecia containing asci with ascospores, while for those of $C$. karstii only conidia were found (Fig. 2).

PCR amplification, sequencing and phylogenetic analysis. Amplicons of approximately 260, 500 and 250 bases were amplified for the ACT, BTUB2 and GAPDH genes regions, respectively, in all isolates (Table 1).

The three sequence data sets of species belonging $C$. boninense and $C$. gloeosporiodes species complex, did not show major conflicts in tree topology, indicating that the three genes could be combined. The multi-gene analysis consisted of 905 characters (including alignment gaps), of which 216 characters were parsimony-informative. MP analysis resulted in three most parsimonious trees $(\mathrm{TL}=$ 369 step, $\mathrm{CI}=0.94, \mathrm{RI}=0.99$ ).
Two main clades are recognized in the phylogenetic tree drawn from the multi-gene analysis. One clade is composed of species included in the $C$. gloeosporioides species complex and most of the Uruguayan isolates resolved as $C$. fructicola (41 isolates). The other main clade is composed of species included in the $C$. boninense species complex and five Uruguayan isolates identified as $C$. karstii. The main clades and sub-clades bootstrap support, robustly resolved all Colletotrichum species for both methods of analysis (Fig. 3). The isolates identified as C. fructicola belong to the same haplotype, except L48 that differs in one nucleotide in the BTUB2 region. With regard to C. karstii three haplotypes were found, L55 and L62, L59 and L65 with two different nucleotides in the GAPDH region and L51 with one different nucleotide in the BTUB2 region.

Pathogenicity tests. Both conidia of $C$. karstii and ascospores of $C$. fruticola, caused typical GLS symptoms in apple leaves and fruits (Table 2). No symptoms were observed in control treatments (Fig. 4A).

The first symptoms consisted in purple to ochre necrotic spot-like lesions on leaves and were already visible three and seven days after inoculation with C. fructicola (Fig. 4B and C) and C. karstii, respectively. Lesions in leaves inoculated with $C$. fructicola evolved rapidly to larger, irregular shape and necrotic lesions at seven days from inoculation

Table 2. Result of pathogenicity test of Colletotrichum fructicola and C. karstii isolates on apple leaves cv. Galaxy and apple fruits cv. Cripps pink

\begin{tabular}{|c|c|c|c|c|c|c|}
\hline Specie & Isolate & $\begin{array}{l}\text { Spore } \\
\text { type }\end{array}$ & $\begin{array}{c}\text { Tissue } \\
\text { inoculated }\end{array}$ & $\begin{array}{c}\text { Initial symptoms } \\
\text { (days from inoculation) }\end{array}$ & $\begin{array}{l}\text { Defoliation } \\
\text { (10 days after } \\
\text { inoculation) }\end{array}$ & $\begin{array}{c}\text { Presence of } \\
\text { acervuli } \\
\text { on leaves }\end{array}$ \\
\hline & & & detached leaves & 3 & & yes \\
\hline \multirow[t]{15}{*}{ C. fructicola } & L49 & ascospores & detached branches & 3 & severe & yes \\
\hline & & & fruit & 21 & & \\
\hline & & & detached leaves & 3 & & yes \\
\hline & L53 & ascospores & detached branches & 3 & severe & yes \\
\hline & & & fruit & 21 & & \\
\hline & & & detached leaves & 3 & & yes \\
\hline & L64 & ascospores & detached branches & 3 & severe & yes \\
\hline & & & fruit & 21 & & \\
\hline & & & detached leaves & 3 & & yes \\
\hline & F71 & ascospores & detached branches & 3 & severe & yes \\
\hline & & & fruit & 21 & & \\
\hline & & & detached leaves & 3 & & yes \\
\hline & F84 & ascospores & detached branches & 3 & severe & yes \\
\hline & & & fruit & 21 & & \\
\hline & & & detached leaves & 7 & & no \\
\hline \multirow[t]{2}{*}{ C. karstii } & L51 & conidia & detached branches & 7 & slight & no \\
\hline & & & fruit & 21 & & \\
\hline
\end{tabular}




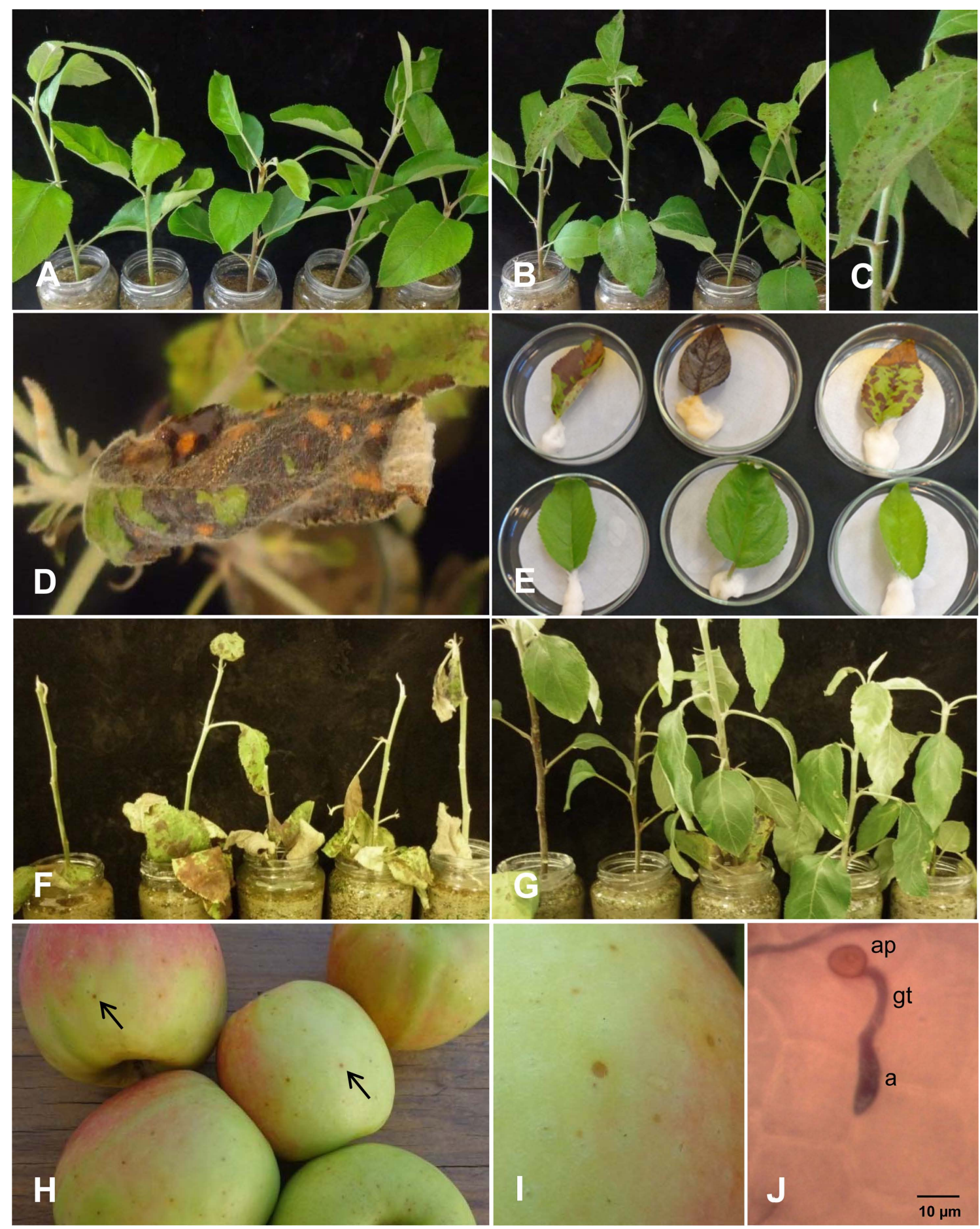

Fig. 4. Pathogenicity tests on twigs and leaves of apple cv. Galaxy and fruits cv. Cripps pink. (A) Twigs used as control ten days after inoculation with steril destiled wather. (B) Initial symptom on leaves three days after inoculation with the isolate L49 of Colletotrichum fructicola. (C) Detail of initial symptoms on leaves. (D) Necrotic lesions on leaves and presence abundant acervuli on leaf seven days after inoculation with the isolate F71 of $C$. fructicola. (E) Necrotic lesions on destached leaves seven days after inoculathion with the isolate L53 of C. fructicola (above) and leaves used as control (below). (F) Mild defoliation ten days after inoculation with the isolate L51 of $C$. karstii. (G) Severe defoliation ten days after inoculation with the isolate L49 of C. fructicola. (H) Lesions fruit (black arrows) 21 days after inoculation with the the isolate L53 of C. fructicola. (I) Detail of lesions fruit (J) Ascospore germinated of isolate L49 of $C$. fructicola after 12 hours of inoculation on apple leaf; (a) ascospore, (gt) germination tube and (ap) appressoria. 
(Fig. 4D and E) and to severe defoliation three days later (Fig. 4F). When inoculated with $C$. karstii the defoliation was mild at ten days from the inoculation (Fig. 4G). Abundant acervuli were present in leaf lesions caused by isolates of $C$. fructicola (Fig. 4D), but never were observed in leaf lesions caused by $C$. karstii.

On fruits, symptoms similarly appeared 21 days after inoculation when inoculated with $C$. fructicola or $C$. karstii isolates. The symptoms consist in small corky lesions that did not evolve to rot (Fig. 4H and I). No reproductive structures were observed on these lesions.

Cultural and conidial characteristics of isolates obtained from inoculated leaves and fruits with $C$. karstii, were confirmed on PDA as previously described fulfilling Koch's postulates.

\section{Discussion}

In Uruguay, GLS was recently reported by Casanova et al. (2017) occurring in a small producing area and, to the best of our knowledge, it still remains restricted to the same locality. The reasons why GLS has not yet spread further are unknown. Microclimatic conditions in this locality seem particularly conductive to GLS development and may indeed have helped to create this situation. It is known that GLS requires warm temperatures and high humidity $(\mathrm{Ca}-$ sanova et al., 2017; González and Sutton, 1999; Velho et al., 2015; Wang et al., 2015a). The GLS-infected apple orchards were established in a low area surrounded by windbreaks, hindering ventilation and possibly resulting in better environmental conditions to the pathogen. Furthermore, the affected area is slightly distant from other commercial apple orchards; being the nearest seven kilometers away.

The widespread randomized distribution of symptoms observed throughout trees and orchards where GLS is present, suggests that ascospores may play a pivotal role in the dispersion of this disease. In this work, ascospores were proofed for the first time to be pathogenic to leaves and fruits confirming its potential to cause infections. The frequent presence of acervuli and perithecia containing conidia and ascospores, respectively, in leaf lesions indicates that both types of spores are regularly produced by most strains of Colleotrichum causing GLS. In contrast to asexual spores of Colletotrichum which are rain-splash-dispersed (Perfect et al., 1999; Sutton and Shane, 1983), those sexually formed are wind-dispersed (Sutton and Shane, 1983). Conidia are released by rain splash and carried by water droplets to short distances generating a disease spatial gradient in focus pattern within the orchard. On the other hand, ascopores are ejected up to a small distance and, usu- ally, dispersed by air currents reaching longer distances and leading to a randomized disease distribution throughout the orchard. The occurrence of symptoms in leaves on the top of the apple tree canopy, strongly suggests that infection has happened with ascospores carried by wind.

In this work it was confirmed that Colletotrichum remains alive in the small fruit corky lesions. Cheon et al. (2016) also were able to isolate $C$. gloeosporioides from symptoms in apple fruits comparable to those of GLS. Furthermore, this fungus did not result in rot development, probably because it could not grow out from small cork-like lesions to surrounding healthy tissues of fruits. Interestingly, in this confined situation it did not build any reproductive structure. It seems that apple fruits can active some resistance mechanisms able to avoid fungus growth from this lesion. Although most GLS-isolates do not have the ability to produce fruit rot, there are a few able to do this (Velho et al., 2015). Thus, investigating the reasons why GLS-isolates are in general not able to cause fruit rot would be an exciting topic for future research.

Based on phylogenetic analysis of GAPDH, BTUB and ACT genomic regions and pathogenicity test, $C$. karstii was found as a new species causing GLS in Uruguay. $C$. fructicola had already been reported associated with this disease by Casanova et al. (2017), but in this work appears as the dominant species (89\%). C. fructicola and C. karstii were identified in Brazil as the causal agent of GLS (Velho et al., 2014, 2015) and C. fructicola as one of the two species associated to GLS in China (Wang et al., 2015b).

The results of this and other studies (Rockenbach et al., 2016; Velho et al., 2015) indicate that $C$. fructicola have an extremely aggressive behavior; within three days it causes visible symptoms on leaves inoculated with conidia or ascospores and a few days later severe defoliation. Moreover, under favorable conditions, warm temperatures and high relative humidity, abundant reproductive structures are formed on leaf lesions. In contrast, $C$. karstii develops much more slowly and reproductive structures seldom appear. Likewise, all isolates of $C$. fructicola grown in laboratory produced conidia and perithecia containing ascospores, but not $C$. karstii.

The species of Colletotrichum found in Uruguay causing GLS are similar to those reported in Southern Brazil. This suggests that apple plants imported from Brazil could have introduced the disease in Uruguay. Another hypothesis is that isolates of C. fructicola and C. karstii from Uruguay responsible for GLS have arisen from isolates capable of causing bitter rot. However, $C$. karstii has not been associated with bitter rot disease in Uruguay (Alaniz et al., 2015). Moreover, Uruguayan populations of $C$. fructicola causing 
bitter rot disease were found to be genetically distinct from Brazilian isolates obtained from GLS and bitter rot diseases (Rockenbach et al., 2016). Therefore, new population studies are needed to compare genetic structure of $C$. fructicola populations causing GLS and bitter rot in Uruguay and Brazil. This might help to elucidate the origin of the GLS in Uruguay.

In summary, the consistency and aggressiveness of the symptoms observed in this and other studies (Gonzalez and Sutton, 1999; Sutton and Sanhueza, 1998; Wang et al., $2015 b$ ), indicate the high impact potential of GLS in causing severe losses on susceptible cultivars if GLS spreads out to further apple producing areas in Uruguay. Developing new strategies for GLS management is extremely necessary because control methods have been currently based on regular applications of fungicides during the summer. Probably, the simultaneously production of ascospores and conidia might be, however, difficult due to their distinct and complementary roles in the development of disease epidemics.

\section{References}

Alaniz, S., Hernández, L. and Mondino, P. 2015. Colletotrichum fructicola is the dominant and one of the most aggressive species causing bitter rot of apple in Uruguay. Trop. Plant Pathol. 40:265-274.

Carbone, I. and Kohn, L. M. 1999. A method for designing primer sets for speciation studies in filamentous ascomycetes. Mycologia 91:553-556.

Casanova, L., Hernández, L., Martínez, E., Velho, A. C., Rockenbach, M. F., Stadnik, M. J., Alaniz, S. and Mondino, P. 2017. First report of glomerella leaf spot of apple caused by Colletotrichum fructicola in Uruguay. Plant Dis. 101:834.

Cheon, W., Lee, S. G. and Jeon, Y. 2016. First report on fruit spot caused by Colletotrichum gloeosporioides in Apple (Malus pumila Mill.) in Korea. Plant Dis. 100:210.

Damm, U., Cannon, P. F., Woudenberg, J. H. C., Johnston, P. R., Weir, B. S., Tan, Y. P., Shivas, R. G. and Crous, P. W. 2012. The Colletotrichum boninense species complex. Stud. Mycol. 73:1-36.

González, E. and Sutton, T. B. 1999. First report of glomerella leaf spot (Glomerella cingulata) of apple in the United States. Plant Dis. 83:1074.2

González, E. and Sutton, T. B. 2004. Population diversity within isolates of Colletotrichum spp. causing glomerella leaf spot and bitter rot of apples in three orchards in North Carolina. Plant Dis. 88:1335-1340.

Guerber, J. C., Liu, B., Correll, J. C. and Johnston, P. R. 2003. Characterization of diversity in Colletotrichum acutatum sensu lato by sequence analysis of two gene introns, mtDNA and intron RFLPs, and mating compatibility. Mycologia 95:872-
895.

Hillis, D. and Bull, J. 1993. An empirical test of bootstrapping as a method for assessing confidence in phylogenetic analysis. Syst. Biol. 42:182-192.

Kumar, S., Stecher, G. and Tamura, K. 2016. MEGA7: Molecular evolutionary genetics analysis version 7.0 for bigger datasets. Mol. Biol. Evol. 33:1870-1874.

Larkin, M. A., Blackshields, G., Brown, N. P., Chenna, R., McGettigan, P. A., McWilliam, H., Valentin, F., Wallace, I. M., Wilm, A., Lopez, R., Thompson, J. D., Gibson, T. J. and Higgins, D. G. 2007. Clustal W and Clustal X version 2.0. Bioinformatics 23:2947-2948.

Liu, Y., Li, B., Wang, C., Liu, C., Kong, X., Zhu, J. and Dai, H. 2016. Genetics and molecular marker identification of a resistance to glomerella leaf spot in apple. Hort. Plant J. 2:121125.

MGAP-DIEA. 2016. Agricultural Statistical Yearbook 2016. URL: https://descargas.mgap.gub.uy/DIEA/Documentos compartidos/Anuario2016/DIEA-Anuario2016cd.pdf [12 March 2019].

Paolocci, F., Rubini, A., Granetti, B. and Arcioni, S. 1999. Rapid molecular approach for reliable identification of Tuber spp. ectomycorrhizae. FEMS Microbiol. Ecol. 28:23-30.

Peever, T. L., Canihos, Y., Olsen, L., Ibañez, A., Liu, Y. C. and Timmer, L. W. 1999. Population genetic structure and host specificity of Alternaria spp. causing brown spot of Minneola tangelo and rough lemon in Florida. Phytopathology 89:851860.

Perfect, S. E., Hughes, H. B., O'Connell, R. J. and Green, J. R. 1999. Colletotrichum: model genus for studies on pathology and fungal-plant interactions. Fungal Genet. Biol. 27:186198.

Rockenbach, M. F., Velho, A. C., Gonçalves, A. E., Mondino, P. E., Alaniz, S. M. and Stadnik, M. J. 2016. Genetic structure of Colletotrichum fructicola associated to apple bitter rot and glomerella leaf spot in southern Brazil and Uruguay. Phytopathology 106:774-781.

Sutton, T. B. and Shane, W. W. 1983. Epidemiology of the perfect stage of Glomerella cingulata on apples. Phytopathology 73:1179-1183.

Sutton, T. B. and Sanhueza, R. M. V. 1998. Necrotic leaf blotch of golden delicious - Glomerella leaf spot: A resolution of common names. Plant Dis. 82:267-268.

Taylor, J. 1971. A necrotic leaf blotch and fruit rot of apple caused by a strain of Glomerella cingulata. Phytopathology 61:221224.

Velho, A. C., Stadnik, M. J., Casanova, L., Mondino, P. and Alaniz, S. 2014. First report of Colletrotrichum karstii causing glomerella leaf spot on apple in Santa Catarina State, Brazil. Plant Dis. 98:157.

Velho, A. C., Alaniz, S., Casanova, L., Mondino, P. and Stadnik, M. J. 2015. New insights into the characterization of Colletotrichum species associated with apple diseases in southern Brazil and Uruguay. Fungal Biol. 119:229-244. 
Wang, B., Li, B. H., Dong, X. L., Wang, C. X. and Zhang, Z. F. 2015a. Effects of temperature, wetness duration, and moisture on the conidial germination, infection, and disease incubation period of Glomerella cingulata. Plant Dis. 99:249-256.

Wang, C. X., Zhang, Z. F., Li, B. H., Wang, H. Y. and Dong, X. L. 2012. First report of glomerella leaf spot of apple caused by Glomerella cingulata in China. Plant Dis. 96:912.

Wang, W., Fu, D. D., Zhang, R. and Sun, G. Y. 2015b. Etiology of apple leaf spot caused by Colletotrichum spp. Mycosystema
34:13-25 (in Chinese).

Weir, B. S., Johnston, P. R. and Damm, U. 2012. The Colletotrichum gloeosporioides species complex. Stud. Mycol. 73:115180.

Woudenberg, J. H., Aveskamp, M. M., de Gruyter, J., Spiers, A. G. and Crous, P. W. 2009. Multiple Didymella teleomorphs are linked to the Phoma clematidina morphotype. Persoonia 22:56-62. 